\title{
Distinct Intramolecular Hydrogen Bonding Dictates Antimicrobial Action of Membrane-targeting Amphiphiles
}

Madhurima Mitra, ${ }^{1, a}$ Mohammad Asad, ${ }^{1, a}$ Sandeep Kumar, ${ }^{1,2}$ Kavita Yadav, ${ }^{1,2}$ Sarika Chaudhary, ${ }^{3}$ Neel Sarovar Bhavesh, ${ }^{4}$ Syma Khalid, ${ }^{5}$ Lipi Thukral, ${ }^{3,{ }^{*}}$ and Avinash Bajaj1,,

1. Laboratory of Nanotechnology and Chemical Biology, Regional Centre for Biotechnology, NCR Biotech Science Cluster, $3^{\text {rd }}$ Milestone, Faridabad-Gurgaon Expressway, Faridabad121001, Haryana, India.

2. Manipal Academy of Higher Education, Manipal-576104, Karnataka, India.

3. CSIR-Institute of Genomics and Integrative Biology, Mathura Road, New Delhi-110025, India.

4. International Centre for Genetic Engineering and Biotechnology, Aruna Asaf Ali Marg, New Delhi-110067, India.

5. School of Chemistry, University of Southampton, Highfield, Southampton-SO17 1BJ, United Kingdom.

a: authors contributed equally.

\section{Corresponding Authors:}

Lipi Thukral, Email: lipi.thukral@igib.res.in

Avinash Bajaj, Email: bajaj@rcb.res.in 


\section{Abstract}

As mechanisms underpinning the molecular interactions between membrane-targeting antimicrobials and Gram-negative bacterial membranes at atomistic scale remain elusive, we used Cholic Acid (CA)-derived amphiphiles with different hydrophobicity as model antimicrobials and assessed the effect of their conformational flexibility on antimicrobial activity. Relative to other hydrophobic counterparts, compound with hexyl chain (6) showed strongest binding with lipopolysaccharide (LPS) of Gram-negative bacterial membranes and acted as an effective antimicrobial. Biomolecular simulations, validated by complimentary approaches, revealed that specific intra-molecular hydrogen-bonding imparts conformationally rigid character to compound $\mathbf{6}$. This conformational stability of compound $\mathbf{6}$ allows minimum but specific interactions of the amphiphile with LPS that are a sum of exothermic processes like electrostatic interactions, membrane insertion and endothermic contributions from disaggregation of LPS. Therefore, our study reveals that membranetargeting mechanism with the help of conformationally selective molecules offers a roadmap for developing future therapeutics against bacterial infections.

Keywords: MD Simulations, Gram-negative Bacteria, Cholic Acid, Amphiphiles, Antibacterial 


\section{Table of Contents Graphic}

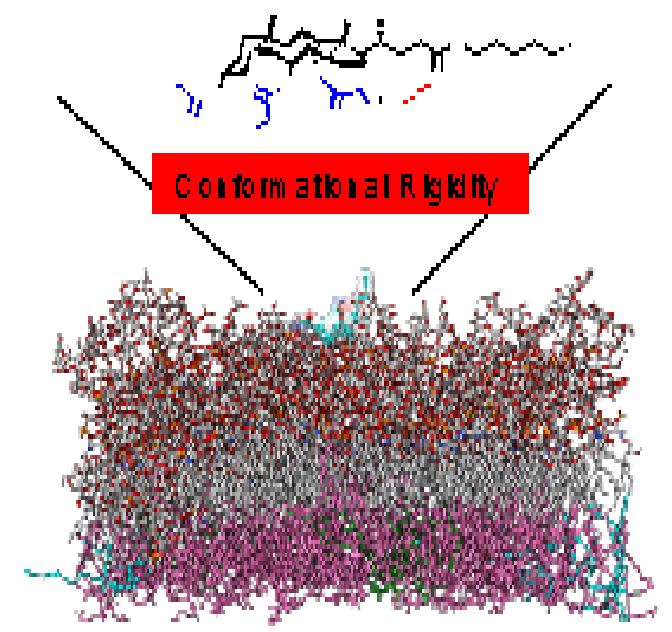




\section{Manuscript Text}

Naturally occurring biomolecules like RNA, proteins and antimicrobial peptides (AMPs) adopt peculiar three-dimensional conformation for executing their functions in cell signaling, enzymatic and other cellular processes. ${ }^{1}$ This natural design of biomolecules has encouraged the engineering of synthetic oligomers with specific conformations that are biologically active over unstructured polymers. ${ }^{2}$ For example, polymeric scaffold based on 4,6-dicarboxy pyridine with increased intramolecular $\mathrm{H}$-bonding mimic three-dimensional conformation of naturally occurring AMPs and possess membrane-targeting antibacterial activities. ${ }^{3}$ Similarly, conformationally constrained arylamide foldamers with basic side chains are more active over less rigid structures that need more basic side chains for required interactions with bacterial membranes. ${ }^{4}$ Recently, engineering and antibacterial screening of Kiadins, artificial variants of AMPs validated by computational algorithm, revealed that peptides enriched in Glycine residues are less effective due to conformational flexibility. ${ }^{5}$

Due to emergence of multi-drug resistance in Gram-negative bacteria, there is a great surge in development of membrane-targeting amphiphiles, ${ }^{6}$ peptides, ${ }^{7}$ lipopeptides, ${ }^{8}$ steroidal amphiphiles ${ }^{9}$ mimicking the facial amphiphilic character of AMPs as effective antimicrobials. ${ }^{10}$ Intricate molecular structure of Gram-negative bacterial membranes present a tough challenge for membrane-targeting amphiphiles to have effective interactions for membrane disruptions. ${ }^{11}$ Lipopolysaccharide (LPS) of Gram-negative bacteria is composed of lipid A linked to O-antigen through a core oligosaccharide spacer. ${ }^{12}$ Intermolecular bridge interactions mediated by $\mathrm{Mg}^{+2}$ and $\mathrm{Ca}^{+2}$ ions of lipid $\mathrm{A}$ molecules make effective permeability barrier for antimicrobials, proteases and hydrophobic drugs. ${ }^{12}$ Therefore, antimicrobial- 
assisted disruptions of outer membrane are less energetically favorable as compared to phospholipid membranes, as it need to break the cation-cross-linked LPS membrane.

Cholic Acid (CA)-derived membrane targeting amphiphiles are known to mimic the facial amphiphilic character of naturally occurring AMPs and act as effective antimicrobials against broad range of bacteria. ${ }^{13-14}$ As critical balance of charge and hydrophobicity is essential for these membrane-targeting antimicrobials to execute distinct membrane disruptions; we raised few critical questions: a) what are the fine-tuning features in terms of charge and hydrophobicity required for effective membrane-targeting antimicrobial action; b) what is the role of conformational rigidity at the LPS membrane interface in antimicrobial activity underlying these amphiphiles with similar chemical functional groups albeit with differential hydrophobicity and c) what is the trade-off between physicochemical attributes such as electrostatic and hydrophobic interactions of these amphiphiles with LPS membranes that is responsible for membrane disruptions. Therefore, we investigated the interactions of CAderived amphiphiles having similar functional groups but different hydrophobicity with complex outer membrane of Gram-negative bacteria that is composed of lipid A linked to Oantigen through a core oligosaccharide spacer.

In this study, we used CA-derived amphiphiles where C-24 carboxylic acid is modified with different hydrophobic alkyl chains, and cationic charge in the form of three glycine residues is attached at hydroxyl termini (Figure S1A). ${ }^{15}$ Antibacterial activities of these amphiphiles against Gram-negative Escherichia coli bacteria showed that compound 6 with hexyl chain is most active with $\mathrm{MIC}_{99}$ of $4.0 \mu \mathrm{M}$ (Figure 1A, S1B). However, further increase in chain length diminished the antibacterial activity of amphiphiles (Figure S1B). We confirmed the membrane-mediated disrupting antibacterial action of compound $\mathbf{6}$ against $E$. coli by time kill 
assay (Figure S1C), followed by $N$-phenyl-1-naphthylamine based fluorescence assay that showed increase in fluorescence on compound 6 treatment (Figure S1D). ${ }^{16}$ Propidium iodide (PI) uptake assay finally confirmed the time and dose-dependent disruption of the bacterial membranes as we observed an increase in number of PI-positive cells (Figure S1E).

We then selected three amphiphiles, compound $1\left(\mathrm{MIC}_{99}=64.0 \mu \mathrm{M}\right), 6(\mathrm{MIC} 99=4.0 \mu \mathrm{M})$ and 9 ( MIC $\left._{99}>256.0 \mu \mathrm{M}\right)$ with varied alkyl chains and diverse antibacterial activity, and investigated the molecular mechanism underlying the effective antimicrobial action of compound 6 over other amphiphiles 1 and 9 (Figure 1A). First, we assessed the binding affinities of compound 1, 6, and $\mathbf{9}$ with LPS using Dansyl-labelled Polymyxin B displacement assay that witnessed strongest affinity of compound $\mathbf{6}$ for LPS among all the amphiphiles (Figure 1B). ${ }^{17}$ Similarly, titration of amphiphiles with Boron-dipyrromethene labelled LPS (BODIPY-LPS) confirmed the strongest affinity of compound 6 with LPS leading to disintegration of LPS aggregates and increase in fluorescence (Figure 1C). ${ }^{18} \mathrm{~A}$ similar trend was observed during anisotropy measurements where amphiphiles were incubated with diphenylhexatriene (DPH)-doped model Gram-negative bacterial membranes, ${ }^{19}$ and we noticed compound 6 being most profound in disrupting the rigidity of the membranes as compared to 1 and 9 (Figure 1D). Dye release assay confirmed that the compound 6mediated destabilization of membranes and maximum release of Fluorescein dextran-4 kDa (FD-4) from liposomes (Figure S2A). ${ }^{20}$ To validate these compound 6-LPS interactions, we assessed the activity of compound $\mathbf{6}$ in presence of LPS and observed dose-dependent inhibition of compound 6-induced PI uptake by the bacteria (Figure S2B).

Binding affinities of compounds 1 and $\mathbf{6}$ (compound 9 was insoluble in water) with model Gram-negative lipid monolayers were then assessed using Surface Plasmon Resonance 
(SPR) studies. ${ }^{21}$ We witnessed strong irreversible interactions of compound $\mathbf{6}$ as compared to poor and reversible binding of compound 1 at the membrane surface (Figure S2C, S2D). The binding constants $\left(K_{1}\right)$ of the first step for compound $\mathbf{1}$ and $\mathbf{6}$ were comparable reflecting their similar electrostatic interactions with bacterial membranes (Figure S2E). Higher affinity of compound $\mathbf{6}$ compared to $\mathbf{1}$ is the result of second step $\left(K_{2}\right)$ that involves integration of hydrophobic tails in the membranes. ${ }^{21}$ Slower ( 1700 fold) dissociation at the second step $\left(k_{\mathrm{d} 2}\right)$ for compound 6 ensued its $>25$-fold stronger overall affinity $\left(K_{\mathrm{A}}\right)$ as compared to compound 1 (Figure S2E). Therefore, tighter association of compound 6 with hydrophobic core of the membrane is facilitated by hexyl side chain as compared to methyl group in compound 1.

Thermodynamics of amphiphile-LPS interactions probed using isothermal titration calorimetry suggested that compound 1 and 6 exhibit endothermic peaks due to disaggregation of LPS followed by prominent exothermic peaks reflecting the actual process of amphiphile-LPS interactions (Figure S3A-B). Compound 6-LPS interactions witnessed enhanced exothermic reaction as compared to compound 1-LPS interactions. ${ }^{22}$ Effect of these interactions were further probed using differential scanning calorimetry (DSC) of model Gram-negative membranes incubated with compounds. Compound 6 induced maximum broadening of phase transition over compound $\mathbf{1}$ and $\mathbf{9}$ with asymmetric endotherm having two overlapping thermal events (Figure S3C). Sharp and broad components of compound $\mathbf{6}$ induced dual transition suggesting the formation of compound 6-poor and compound 6-enriched membrane domains that might be responsible for membrane perturbations. ${ }^{23}$

Above mentioned biophysical studies therefore confirmed strong interactions of compound 6 with LPS and model Gram-negative membranes that are responsible for its effective 
antimicrobial activity. To examine these molecular interactions in atomistic detail, we performed molecular dynamics (MD) simulations of compounds $(\mathbf{1}, \mathbf{6}$, or $\mathbf{9})$ with model Gramnegative bacterial membranes.

Our model membranes consist of LPS molecules in the outer leaflet and varied phospholipids in inner leaflet to simulate the physiologically relevant bacterial membrane interface (Figure S4). ${ }^{24}$ In contrast to simplistic phospholipid membrane models, LPS membrane model provides a robust platform to study intricate and complex interactions of amphiphile at bacterial membrane interface..$^{25}$ The three-dimensional structures of compound $\mathbf{1 ,} \mathbf{6}$ and $\mathbf{9}$, shown in supplementary figure S5A, were parameterized individually by ATB topology server. For studying amphiphile-membrane interactions, we placed the amphiphiles away from the membrane to capture unbiased interactions with the hydrophilic LPS molecules (Figure S5B). At the end of the simulation ( $\sim 500 \mathrm{~ns})$, we observed marked differences in the membrane architecture along with simultaneous interactions with membrane lipids (Figure S5C). Membrane thickness measurements caused by each amphiphile witnessed significant thinning of membranes by compound $\mathbf{6}$, in comparison to 1 and $\mathbf{9}$. We observed compound $\mathbf{6}$ induced structural changes in the range of $\sim 1 \mathrm{~nm}$ as opposed to overall $4-5 \mathrm{~nm}$ slab in control simulation (Figure 2A). The membrane geometrical changes were also correlated with probability density maps of compound location as a function of $X Y$ axis of the system suggesting that precise interactions of molecule location with LPS lipids results in greater membrane disturbance (Figure S5D). These changes confirmed clear structural impact of compound 6 on LPS membranes with sparse areas that demonstrated distributed and perturbed lipid arrangement (Figure 2A). 
Given the fact that membrane perturbations introduced by compound 6 will be stoichiometrically dependent; we systematically explored additional MD simulations with higher concentration of compound 6 (5 and 20 molecules) (Figure S6A, S6B). The time evolution of structural changes witnessed drastic membrane perturbations with complete loss of membrane integrity (Figure S6C, S6D). Membrane changes were quantified, and significant membrane thinness compared to the control membrane was observed. Interestingly, crowded simulations showed permeation of compound 6 across the membranes suggesting the ability of compound 6 to cross high energetic barriers at LPS interface (Figure S7A). In fact, the molecules in higher concentration were observed to interact with phospholipid inner leaflet (Figure S7B).

Above observations prompted us to investigate the conformational preferences of the amphiphiles and their specific interactions with LPS membranes. MD trajectories were analyzed for conformational flexibility of compound 1, $\mathbf{6}$ and $\mathbf{9}$ during their association with LPS membranes. Variability of 5000 conformations for each amphiphile in MD simulations was determined by calculating root mean square fluctuations (RMSF) and these calculations were averaged throughout the simulation time. Figure 2B displayed the mapped RMSF or the flexibility values of each atom that enabled us to localize the regions with highest mobility. Compound $\mathbf{9}$ with longest hydrophobic chain showed highest mobility followed by compound $\mathbf{1}$ and $\mathbf{6}$. Compound $\mathbf{6}$, to our surprise, did not show any flexibility and remained in a specific conformation.

To better understand the individual conformational space of each amphiphile, we computed and extracted clusters of amphiphiles during the entire MD trajectory. One major cluster for compound 1 (58.4\%), compound 6 (98\%) and compound 9 (65.4\%) was found throughout 
the simulations (Figure S8). Superimposed conformations of amphiphiles extracted from MD trajectories across different time points witnessed clear variations in molecular arrangement of compound $\mathbf{1}$ and $\mathbf{9}$, while compound $\mathbf{6}$ remained in distinct inflexible state (Figure $\mathbf{2 C}$ ). We also probed configurational state of CA backbone by monitoring angles between representative atoms (Figure S9A-B). The trajectories of compound 6 displayed narrow peak indicating the confined space relative to other amphiphiles (Figure S9C-D). Therefore, compound 1 and 9 explored more phase space while bound to the membranes and in contrast, compound $\mathbf{6}$ was confined to relatively rigid conformational space.

To assess the molecular interactions of these amphiphiles with membranes in atomistic detail, we divided the amphiphiles into three chemical segments, glycine, CA and hydrophobic tail. We then probed the time evolution of distance based membrane contacts (any contact within $\delta 0.5 \mathrm{~nm}$ of membrane atom) of these amphiphiles with LPS and observed that compound 6 executed least number of membrane contacts in all the segments (Figure S10). Towards the end of simulations, CA backbone in compound 6 showed $\sim 10-20$ membrane contacts and other amphiphiles showed $>60$ contacts.

As these amphiphile-membrane contacts may include several weak non-covalent bonds including hydrogen bonding, we examined the exact nature of these dominating intermolecular interactions. Surprisingly, compound $\mathbf{1}$ and $\mathbf{9}$ showed maximum number of contacts with LPS through hydrogen-bonding as opposed to compound 6 that is bound to the membrane surface in a highly specific manner (Figure 3A). Nature and significance of hydrogen bonding pattern throughout the trajectory arises from protons of glycine nitrogen that make stable hydrogen bonding with oxygen of LPS sugar parts (Figure 3B). Analysis of compound 6-LPS interactions in atomistic level revealed that only amine protons of two 
glycine units (attached at C-3 and C-7 of CA) are accessible for intermolecular $\mathrm{H}$-bonding (Figure 3B) as compared to amine protons of three glycine units in case of compound $\mathbf{1}$ and 9 (Figure S11). We observed that amine protons of third glycine unit (attached at C-12 of CA) in case of compound 6 exhibit one intramolecular hydrogen bond with C-24 carbonyl group providing the conformational rigidity to the amphiphile (Figure 3C, 3D).

Next, we set out to validate the confirmation and intramolecular $\mathrm{H}$-bonding as depicted by MD simulations using ${ }^{1} \mathrm{H}$ NMR studies. We therefore performed $1 \mathrm{H}$ NMR of $\mathbf{1}, \mathbf{6}$ and $\mathbf{9}$ in DMSO- $\mathrm{d}_{6}$ and $\mathrm{CDCl}_{3}$ as intramolecular $\mathrm{H}$-bonded protons are usually less sensitive to changes in solvent composition as compared to intermolecular $\mathrm{H}$-bonded protons. ${ }^{26}$ In DMSO-d6, glycine amine protons of $6(10.4 \mathrm{~Hz})$ had narrow linewidth (Full width at half maximum) at $\delta 8.669 \mathrm{ppm}$ as compared to $1(24.73 \mathrm{~Hz}$ at $\delta 8.608 \mathrm{ppm})$ and $\mathbf{9}(23.67 \mathrm{~Hz}$ at d $8.473 \mathrm{ppm}$ ) suggesting the rigid conformation of 6 (Figure $4 \mathrm{~A}$ ). In $\mathrm{CDCl}_{3}$, we observed a broad peak for glycine amine protons of compound 1 (at $\delta 8.761 \mathrm{ppm}$ ) and 9 (at $\delta$ 8.37-8.52 ppm) as compared to that in DMSO-d6 suggesting that all amine protons in $\mathbf{1}$ and $\mathbf{6}$ are solvent sensitive and do not perform any intramolecular H- bonding (Figure 4B). In contrast, we observed two sharp peaks at $\delta 8.385$ and $8.954 \mathrm{ppm}$ for three and six amine protons that confirmed that glycine amine protons in case of compound $\mathbf{6}$ are less sensitive to solvent and molecule retains its rigid conformation (Figure 4B).

Intramolecular $\mathrm{H}$-bonding in case of $\mathbf{6}$ would bring protons of hydrophobic side chain close to amine protons of glycine residue attached at $\mathrm{C}-12$, and we can observe nOe cross peaks between amine protons of glycine and CA backbone. Therefore, we performed $2 \mathrm{D}\left[{ }^{1} \mathrm{H},{ }^{1} \mathrm{H}\right]$ TOCSY and $\left[{ }^{1} \mathrm{H},{ }^{1} \mathrm{H}\right]$ NOESY studies. Amine proton showed nOe cross peaks with protons of 
hydrophobic bile acid backbone at $25^{\circ} \mathrm{C}$ confirming the intramolecular $\mathrm{H}$ - bonding of amine protons with carbonyl oxygen at C-24 position (Figure 4C, black contours). These nOe cross peaks disappeared at $50^{\circ} \mathrm{C}$ possibly due to weakening of $\mathrm{H}$-bond at higher temperature (Figure 4C, red contours). Absence of these nOe cross peaks in compound $\mathbf{1}$ and $\mathbf{9}$ confirmed absence of intramolecular H-bonding (Figure 4D). These results suggests that a specific alkyl chain length at C24-carboxyl group of CA allows the compound 6 to fold for intramolecular $\mathrm{H}$-bonding that is not possible in compound $\mathbf{1}$ due to short alkyl chain and in compound 9 due to highly flexible long chain.

In summary, our studies elucidated that conformational rigidity of compound 6 through intramolecular hydrogen bonding and its specific interactions with LPS is essential for its antimicrobial action. The conceptual framework presented here for specific attributes of membrane targeting amphiphiles and understanding of the molecular processes involved in interactions of these amphiphiles with model bacterial membranes can help in design of future antimicrobial agents.

Supporting information: Supporting information is available on www.pubs.acs.org Supplementary figures S1-S11, Tables S1-S2, and experimental section (PDF).

Acknowledgements: We thank RCB and IGIB for intramural funding, and Department of Biotechnology, Govt. of India for funding this project (BT/PR12297/MED/29/895/2014 and BT/PR17525/MED/29/1021/2016). S.K. thanks RCB for research fellowship. Financial support from the DBT-RA Program in Biotechnology and Life Sciences is gratefully acknowledged. LT thanks intramural CSIR grant MLP 1805 for funding this study at CSIRIGIB. We also acknowledge CSIR-4PI for supercomputing resources.

Conflicting interests: Authors declare no conflicting interests. 


\section{References}

1. Hill, D. J.; Mio, M. J.; Prince, R. B.; Hughes, T. S.; Moore, J. S. A Field Guide to Foldamers. Chem. Rev. 2001, 101, 3893-4012.

2. Violette, A.; Fournel, S.; Lamour, K.; Chaloin, O.; Frisch, B.; Briand, J. P.; Monteil, H.; Guichard, G. Mimicking Helical Antibacterial Peptides with Nonpeptidic Folding Oligomers. Chem. Biol. 2006, 13, 531-538.

3. Tang, H.; Doerksen, R. J.; Jones, T. V.; Klein, M. L.; Tew, G. N. Biomimetic Facially Amphiphilic Antibacterial Oligomers with Conformationally Stiff Backbones. Chem. Biol. 2006, 13, 427-435.

4. Choi, S.; Isaacs, A.; Clements, D.; Liu, D.; Kim, H.; Scott, R. W.; Winkler, J. D.; DeGrado, W. F. De Novo Design and in vivo Activity of Conformationally Restrained Antimicrobial Arylamide Foldamers. Proc. Natl. Acad. Sci. 2009, 106, 6968 -6973

5. Rončević, T.; Vukičević, D.; Ilić, N.; Krce, L.; Gajski, G.; Tonkić, M.; Goić-Barišić, I.; Zoranić, L.; Sonavane, Y.; Benincasa, M.; Juretić, D.; Maravić, A.; Tossi, A. Antibacterial Activity Affected by the Conformational Flexibility in Glycine-Lysine Based a-Helical Antimicrobial Peptides. J. Med. Chem. 2018, 61, 2924-2936.

6. Hoque, J.; Konai, M. M.; Sequeira, S. S.; Samaddar, S.; Haldar, J. Antibacterial and Antibiofilm Activity of Cationic Small Molecules with Spatial Positioning of Hydrophobicity: An in Vitro and in Vivo Evaluation. J. Med. Chem. 2016, 59, 10750-10762.

7. Niu, Y.; Wang, M.; Cao, Y.; Nimmagadda, A.; Hu, J.; Wu, Y.; Cai, J.; Ye, X. S. Rational Design of Dimeric Lysine N-Alkylamides as Potent and Broad-Spectrum Antibacterial Agents. J. Med. Chem. 2018, 61, 2865-2874.

8. Hamley, I. W. Lipopeptides: From Self-Assembly to Bioactivity. Chem. Commun. 2015, 51, 
8574-8583.

9. Bansal, S.; Singh, M.; Kidwai, S.; Bhargava, P.; Singh, A.; Sreekanth, V.; Singh, R.; Bajaj, A. Bile Acid Amphiphiles with Tunable Head Groups as Highly Selective Antitubsercular Agents. MedChemComm 2014, 5, 1761-1768.

10. Abdel Monaim, S. A. H.; Ramchuran, E. J.; El-Faham, A.; Albericio, F.; de la Torre, B. G. Converting Teixobactin into a Cationic Antimicrobial Peptide (AMP). J. Med. Chem. 2017, 60, $7476-7482$.

11. Rietschel, E. T.; Kirikae, T.; Schade, F. U.; Mamat, U.; Schmidt, G.; Loppnow, H.; Ulmer, A. J.; Zähringer, U.; Seydel, U.; Di Padova, F. Bacterial Endotoxin: Molecular Relationships of Structure to Activity and Function. FASEB J. 1994, 8, 217-225.

12. Meredith, T. C.; Aggarwal, P.; Mamat, U.; Lindner, B.; Woodard, R. W. Redefining the Requisite Lipopolysaccharide Structure in Escherichia coli. ACS Chem Biol. 2006, 1, 33-42.

13. Lai, X. Z.; Feng, Y.; Pollard, J.; Chin, J. N.; Rybak, M. J.; Bucki, R.; Epand, R. F.; Epand, R. M.; Savage, P. B. Ceragenins: Cholic Acid-Based Mimics of Antimicrobial Peptides. Acc. Chem. Res. 2008, 41, 1233-1240.

14. Ding, B.; Yin, N.; Liu, Y.; Cardenas-Garcia, J.; Evanson, R.; Orsak, T.; Fan, M.; Turin, G.; Savage, P. B. Origins of Cell Selectivity of Cationic Steroid Antibiotics. J. Am. Chem. Soc. 2004, 126, 13642-13648.

15. Yadav, K.; Yavvari, P. S.; Pal, S.; Kumar, S.; Mishra, D.; Gupta, S.; Mitra, M.; Soni, V.; Khare, N.; Sharma, P.; Srikanth, C. V.; Kapil, A; Singh, A.; Nandicoori, V. K.; Bajaj, A. Oral Delivery of Cholic Acid-Derived Amphiphile helps in Combating Salmonella-mediated Gut Infection and Inflammation. Bioconjugate Chem. DOI: 10.1021/acs.bioconjchem.8b00880.

16. Loh, B.; Grant, C.; Hancock, R. E. W. Use of the Fluorescent Probe 1-N- 
Phenylnaphthylamine to Study the Interactions of Aminoglycoside Antibiotics with the Outer Membrane of Pseudomonas aeruginosa. Antimicrob. Agents Chemother. 1984, 26, 546-551. 17. Zhang, L.; Dhillon, P.; Yan, H.; Farmer, S.; Hancock, R. E. W. Interactions of Bacterial Cationic Peptide Antibiotics with Outer and Cytoplasmic Membranes of Pseudomonas aeruginosa. Antimicrob. Agents Chemother. 2000, 44, 3317-3321.

18. Uppu, D. S. S. M.; Haldar, J. Lipopolysaccharide Neutralization by Cationic-Amphiphilic Polymers through Pseudo-aggregate Formation. Biomacromolecules 2016, 17, 862-873.

19. Bhargava, P.; Singh, M.; Sreekanth, V.; Bajaj, A. Nature of the Charged Headgroup Determines the Fusogenic Potential and Membrane Properties of Lithocholic Acid Phospholipids. J. Phys. Chem. B 2014, 118, 9341-9348.

20. Sani, M.-A.; Whitwell, T. C.; Gehman, J. D.; Robins-Browne, R. M.; Pantarat, N.; Attard, T. J.; Reynolds, E. C.; O'Brien-Simpson, N. M.; Separovic, F. Maculatin 1.1 Disrupts Staphylococcus aureus Lipid Membranes via a Pore Mechanism. Antimicrob. Agents Chemother. 2013, 57, 3593-3600.

21. Mozsolits, H.; Wirth, H. J.; Werkmeister, J.; Aguilar, M. I. Analysis of Antimicrobial Peptide Interactions with Hybrid Bilayer Membrane Systems Using Surface Plasmon Resonance. Biochim. Biophys. Acta - Biomembr. 2001, 1512, 64-76.

22. Epand, R. F.; Maloy, W. L.; Ramamoorthy, A.; Epand, R. M. Probing the "Charge Cluster Mechanism" in Amphipathic Helical Cationic Antimicrobial Peptides. Biochemistry 2010, 49, 4076-4084.

23. Singh, M.; Bajaj, A. Unraveling the Impact of Hydroxylation on Interactions of Bile Acid Cationic Lipids with Model Membranes by In-Depth Calorimetry Studies. Phys. Chem. Chem. Phys. 2014, 16, 19266-19274. 
24. Jefferies, D.; Hsu, P. C.; Khalid, S. Through the Lipopolysaccharide Glass: A Potent Antimicrobial Peptide Induces Phase Changes in Membranes. Biochemistry 2017, 56, 16721679.

25. Berglund, N. A.; Piggot, T. J.; Jefferies, D.; Sessions, R. B.; Bond, P. J.; Khalid, S. Interaction of the Antimicrobial Peptide Polymyxin B1 with Both Membranes of E. coli: A Molecular Dynamics Study. PLOS Comput. Biol. 2015, 11, e1004180.

26. Yuan, L.; Zeng, H.; Yamato, K.; Sanford, A. R.; Feng, W.; Atreya, H. S.; Sukumaran, D. K.; Szyperski, T.; Gong, B. Helical Aromatic Oligoamides: Reliable, Readily Predictable Folding from the Combination of Rigidified Structural Motifs. J. Am. Chem. Soc. 2004, 126, $16528-16537$. 
A
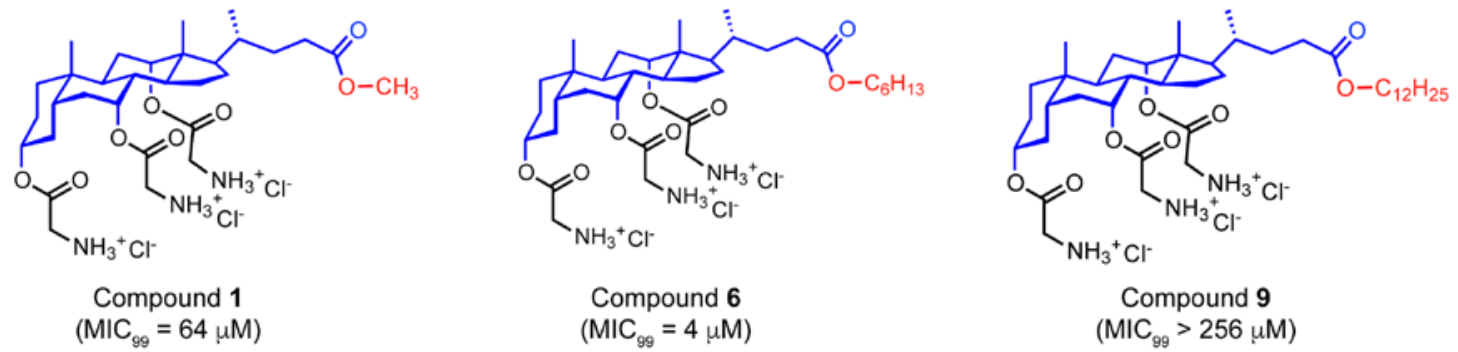

B

C
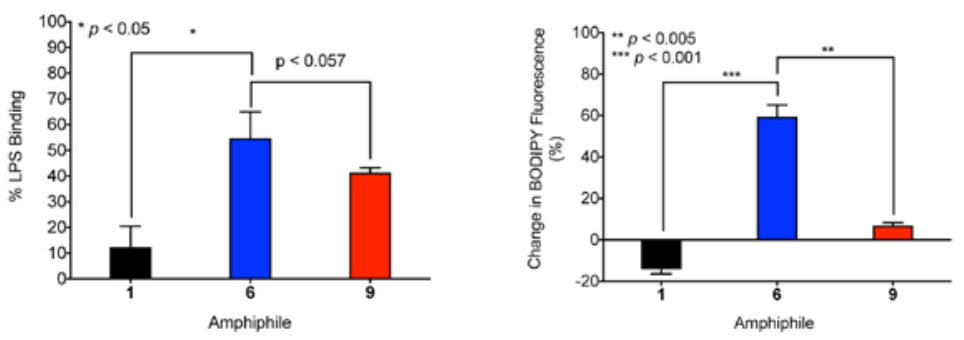

D

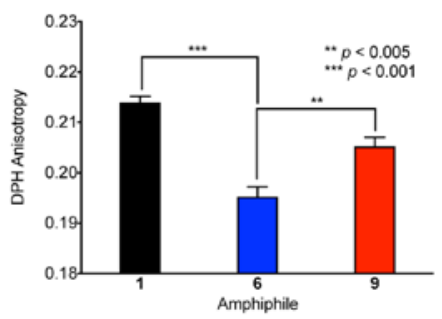

Figure 1. A) Molecular structures of CA-derived amphiphiles (1, $\mathbf{6}$ and $\mathbf{9})$ with their $\mathrm{MIC}_{99}$ against E. coli. B) LPS binding percentage of amphiphiles 1, 6 and 9 using Dansyl Polymyxin B-displacement assay confirm the effective binding ability of $\mathbf{6}$ over $\mathbf{1}$ and $\mathbf{9}$. C) Change in fluorescence of BODIPY-LPS after incubation of BODIPY-LPS aggregates with 1, 6 and 9 amphiphiles show the effective binding of 6 . D) Change in anisotropy of diphenylhexatriene (DPH) in model Gram-negative bacterial membranes on incubation with amphiphiles 1, 6 and $\mathbf{9}$ confirm the ability of $\mathbf{6}$ to perturb bacterial membranes. 

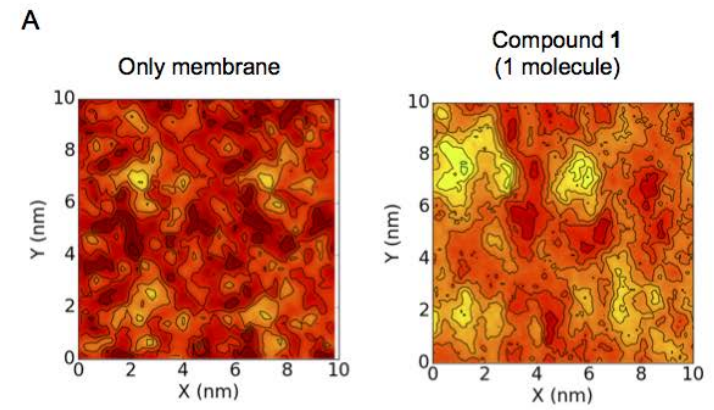

B
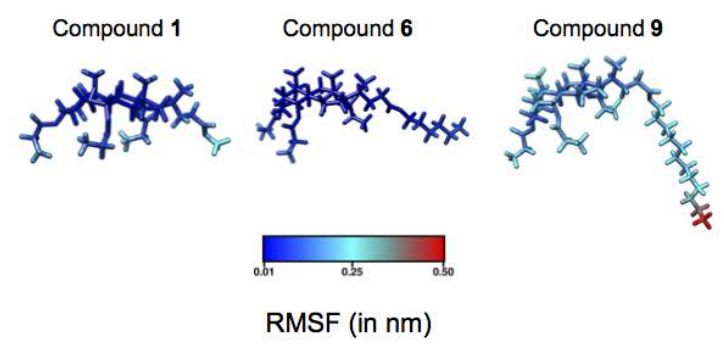
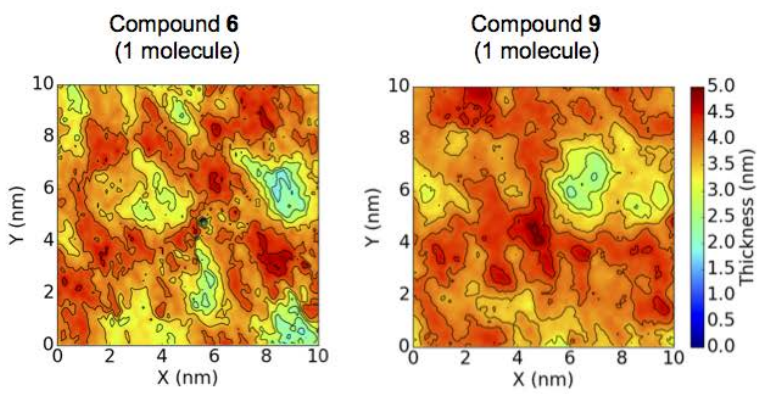

C

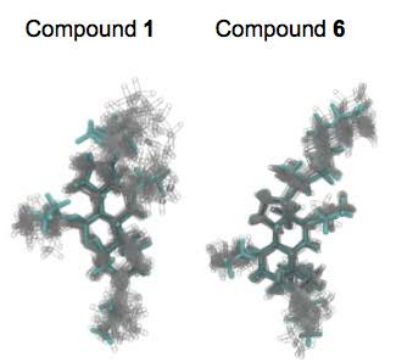

Compound $\mathbf{9}$

Figure 2. A) Contour plots showing membrane thinning on amphiphile-membrane interactions in comparison to control membranes as revealed by MD simulations with yellow color indicating regions with membrane thinning. B) Mapping of root mean square fluctuation (RMSF) as a function of each atom in compound 1, 6 and $\mathbf{9}$ suggest that compound $\mathbf{9}$ has the maximum mobility across all groups, including cholic, amino ester and hydrophobic tail. In contrast, compound $\mathbf{6}$ is least flexible molecule and compound $\mathbf{1}$ shows intermediate behaviour. C) Superimposed conformations of compound 1, 6 and 9 across different time points reveal that compound 6 remain in distinct rigid state. In contrast, compound 1 and 9 has clear variations in their molecular arrangement. The conformations are dumped at several time points (shown in grey) and last snapshot of the entire trajectory (in blue) are shown to represent conformational variability of amphiphiles in membrane bound state. The lipids and water are not shown for clarity. 


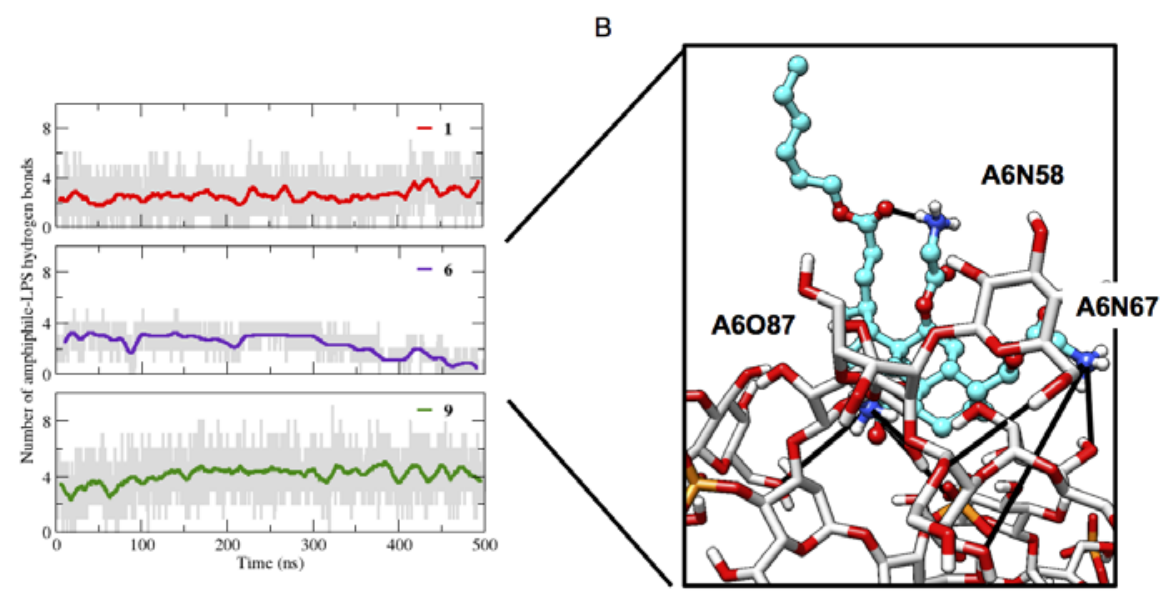

C

D
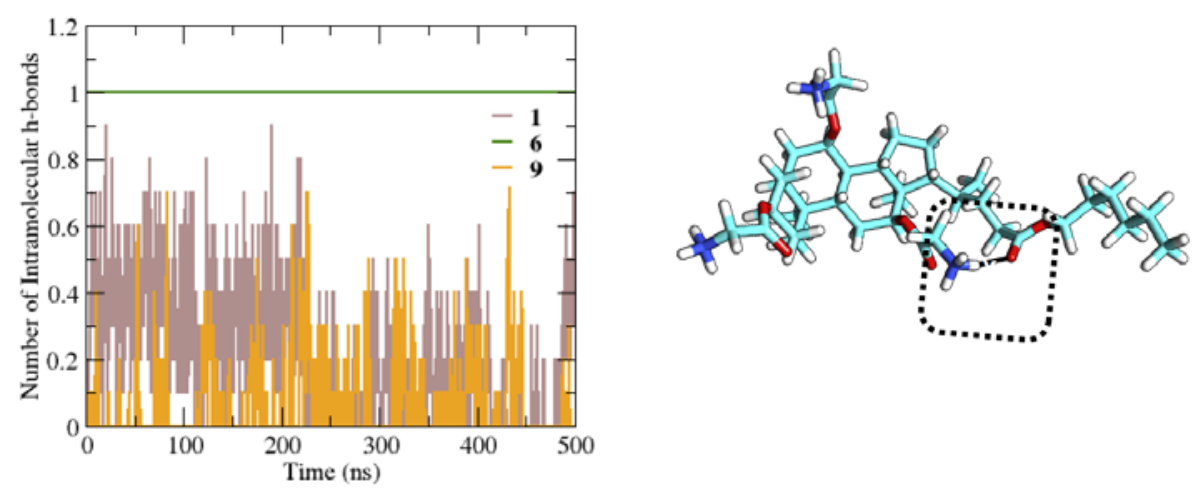

Figure 3. A) Number of inter-molecular hydrogen bonds with LPS membrane as a function of time for compounds 1, 6 and 9 confirm that compound $\mathbf{6}$ showed minimum but specific hydrogen bonding interactions with LPS. B) Representative snapshot showing compound 6-LPS hydrogen bonding with participating atoms highlighted in boxes. Amine protons at A6N58 was unavailable to LPS as it was forming intramolecular bonding. C) Time-evolution of intra-molecular hydrogen bonding in compound $\mathbf{1}, \mathbf{6}$, and $\mathbf{9}$ throughout the timescale. We observed only one intramolecular hydrogen bond within compound 6 based on $0.35 \mathrm{~nm} \mathrm{H}$-bond cut-off. D) Representative snapshots of compound $\mathbf{6}$ showed the formation of intramolecular H-bond between amine protons of glycine attached at C-12 of CA backbone and oxygen of C-24 carbonyl group. 
A

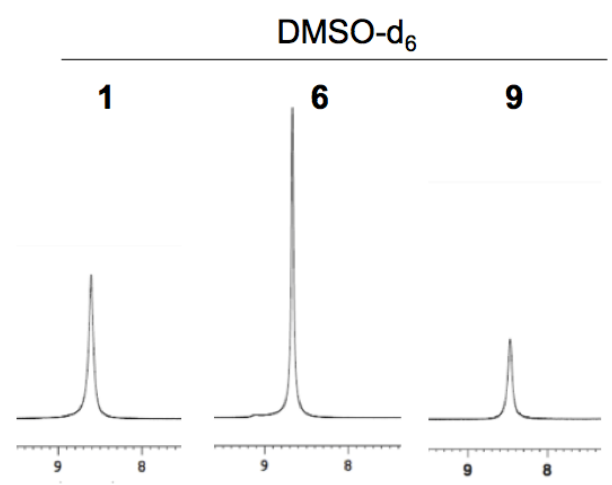

B

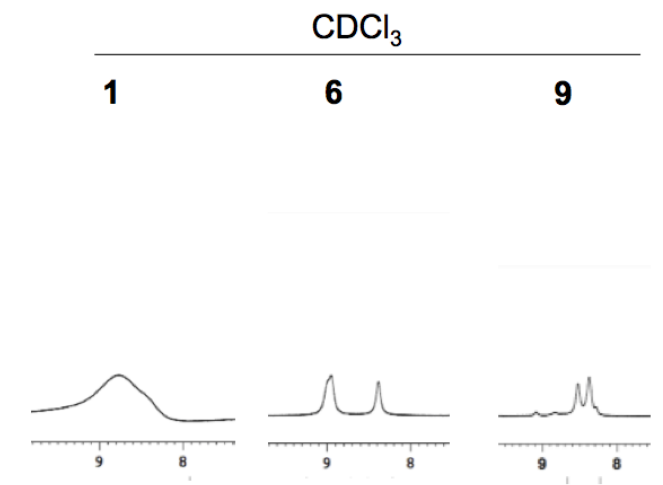

C

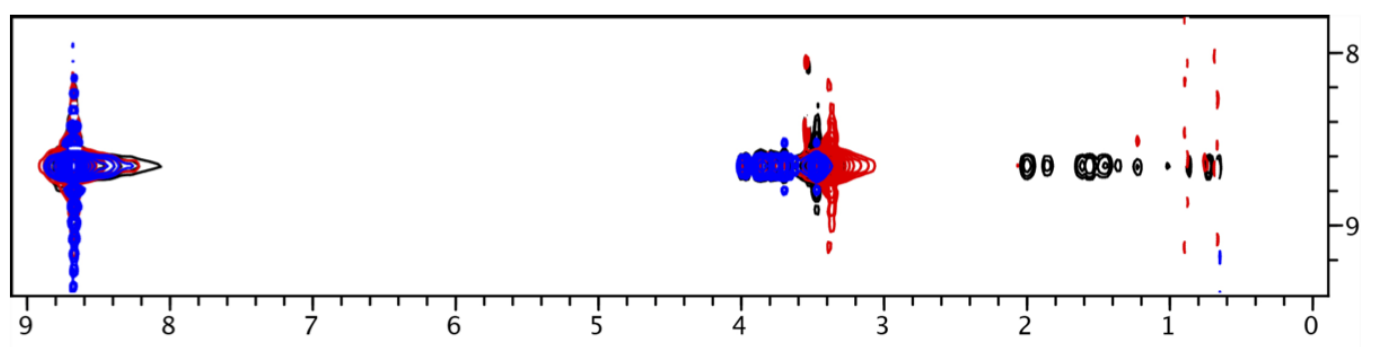

D

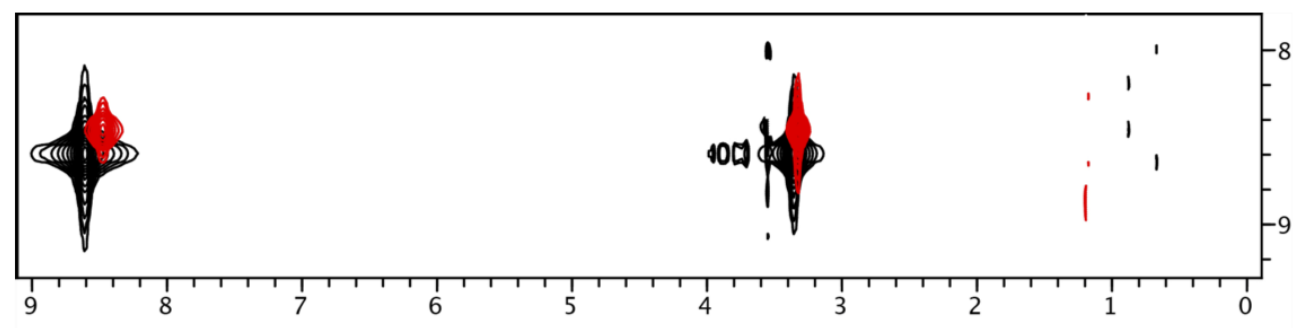

Figure 4. A) ${ }^{1} \mathrm{H}$ NMR spectra of compounds 1, 6 and 9 in DMSO-d 6 show that amine protons of 6 had narrow $(10.4 \mathrm{~Hz}$ ) linewidths (Full width at half maximum) as compared to 1 (24.73 Hz), and 9 $(23.67 \mathrm{~Hz})$ confirming the rigid nature of 6 . B) ${ }^{1} \mathrm{H}$ NMR spectra of $\mathbf{1}, 6$ and 9 in $\mathrm{CDCl}_{3}$ show broadening and shifting of amine protons of $\mathbf{1}$ and $\mathbf{9}$ due to solvent effect. In contrast, 6 showed sharp peaks at d 8.385 and 8.954 ppm due to its less flexible nature. C) 2D $\left[{ }^{1} \mathrm{H},{ }^{1} \mathrm{H}\right]$ NOESY (Black) spectra of 6 shows space correlation of $\mathrm{H}$-bonded amine proton and protons of bile acid backbone in 6 (at region 1-2 ppm) confirming the intramolecular $\mathrm{H}$-bonding. Effect of temperature (Red) on NOESY spectra of 6 reveal the disappearance of the space correlation (nOe cross peaks) that confirm the intramolecular $\mathrm{H}$-bonding. Black: 6 NOESY at $25^{\circ} \mathrm{C}$, Red: NOESY at $50^{\circ} \mathrm{C}, Y^{\prime}$ ellow: TOCSY at $50^{\circ} \mathrm{C}$. D) 2D $\left[{ }^{1} \mathrm{H},{ }^{1} \mathrm{H}\right]$ NOESY spectra of 1 (Black) and 9 (Red) showed no space correlation of amine protons with other protons of CA backbone confirming no intramolecular $\mathrm{H}$ bonding in $\mathbf{1}$ and $\mathbf{9}$. 